\title{
Rekonfigurationstechniken und Anwendungsgebiete für ein programmierbares $\mathbf{G}_{m}$-C Analog-Filter
}

\author{
J. Becker, F. Henrici, and Y. Manoli \\ Universität Freiburg, Institut für Mikrosystemtechnik - IMTEK, Lehrstuhl für Mikroelektronik, Germany
}

\begin{abstract}
Zusammenfassung. FPAAs (Field Programmable Analog Arrays) erlauben es, analoge Signalübertragungsfunktionen auf Hardware abzubilden, die von veränderbaren digitalen Konfigurationsdaten abhängen. Sowohl für das Design von Filterstrukturen auf Systemebene als auch für die genaue Simulation ihrer analogen Übertragungsfunktion auf Transistorebene ist es dabei notwendig, die entsprechenden Konfigurationsdaten einzugeben bevor die Phasen/Betragsanalyse durchgeführt werden kann. Die vorliegende Arbeit stellt ein graphisches Entwurfsprogramm zur Instantiierung von analogen Filtern für ein FPAA mit $17 \mathrm{~g}_{m}$-C Blöcken vor. Es wird über erste Simulationsergebnisse berichtet, die die Machbarkeit mit Eckfrequenzen bis zu hundert Mhz bestätigen. Simulierte Übertragungsfunktionen von grundlegenden Bausteinen werden mit der Theorie verglichen und zu einer exemplarischen Instantiierung eines Butterworth-Filters der vierten Ordnung weitergeführt.
\end{abstract}

\section{Einleitung}

$\mathrm{G}_{m}$-Zellen bilden die Basis für hochfrequente zeitkontinuierliche aktive Filter und finden in adaptiven Filtern für analoge Datenerfassung Verwendung (Pavan and Tsividis, 2000). Eine verallgemeinerte Idee ist die von programmierbaren Analogarrays (FPAAs), wo nicht nur Filterparameter sondern komplette Filterstrukturen rekonfigurierbar sind. In (Becker and Manoli, 2004a) wurde ein FPAA-Design mit rekonfigurierbaren $\mathrm{g}_{m}$-Zellen vorgestellt und die Umsetzung als integrierter Schaltkreis vorgeschlagen. Der Entwurf ist als Bestandteil von Systems-on-Chip gedacht, wo eine digitale Regelung auf Änderungen der Umgebung oder Teilausfälle des Analogteils reagieren kann, um mit einer geeigneten Rekonfiguration die Funktion des Systems aufrechtzuerhalten. Da das Analogarray in den gleichen Standard-CMOS-Prozessen

Correspondence to: J. Becker (jobeck@imtek.de)

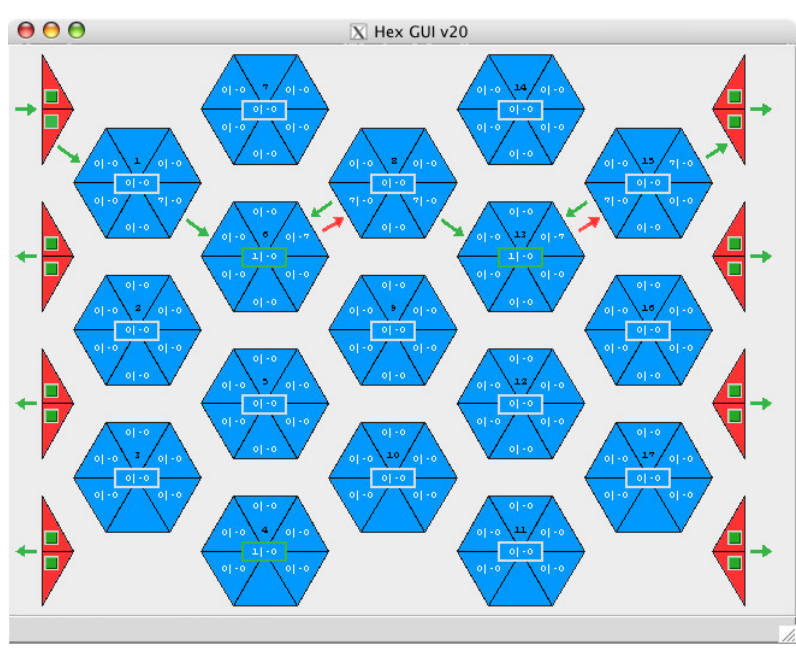

Abbildung 1. GUI des Entwurfs-Programms.

gefertigt werden kann wie der Digitalteil, ist eine nahtlose Integration in Systems-on-Chip möglich.

Eine detailierte Beschreibung des kompletten FPAAEntwurfs überschreitet den Rahmen dieser Arbeit und der Leser wird für eine vollständige Erklärung auf Schaltkreisebene auf (Becker and Manoli, 2004b) verwiesen. Im Prinzip sind 17 konfigurierbare analoge Blöcke (CABs) in einem zweidimensionalen Array mit hexagonaler Struktur angeordnet. Diese ermöglicht sowohl Verschaltungen für parallele und serielle Verbindungen als auch für Signalrückkopplung. Wie in Abb. 1 dargestellt, besitzen alle CABs einstellbare Transkonduktanz-Verstärker ( $\mathrm{g}_{m}$-Zellen), die die Verbindung zur jeweiligen Nachbarzelle herstellen. Die Selbstrückkopplung des $\mathrm{CAB}$ bildet eine zusätzliche einstellbare $\mathrm{g}_{m}$-Zelle und die Eingänge der Zelle sind zu einem Knoten zusammengefasst. Die Parallelschaltung von mehreren Einheitstranskonduktoren erzeugt im Eingangsknoten eine große pa-

Published by Copernicus GmbH on behalf of the URSI Landesausschuss in der Bundesrepublik Deutschland e.V. 
rasitäre Kapazität. Ein Hauptmerkmal dieses Entwurfs ist es, dass diese Kapazität als Integrationskapazität einer $\mathrm{g}_{m}$ C Stufe verwendet wird. Eine $\mathrm{g}_{m}$-C Stufe besteht somit aus einem Zweigtranskonduktor und der parasitären Eingangskapazität der nachfolgenden Nachbarzelle.

Die Simulation des analogen Schaltungsteils auf Transistorebene ist an zwei gewissen Stufen des Entwurfsablaufs eines FPAAs besonders erforderlich: erstens werden während des Schaltungsentwurfs des programmierbaren Arrays Simulationen zur Dimensionierung der $\mathrm{g}_{m}$-Zellen und aller unterstützenden Schaltkreise durchgeführt. Zweitens ist es ratsam, nachdem das FPAA gefertigt wurde, den Frequenzgang eines Filteraufbaus durch Simulation abzuschätzen, bevor er in realer Hardware umgesetzt wird. In beiden Fällen müssen die digitalen Konfigurationsdaten zur Verfügung stehen bevor eine Simulation gestartet werden kann, damit der Zustand der Konfigurationsschalter definiert ist. Im Folgenden wird gezeigt, wie Konfigurationsdaten für programmierbare Systeme während der Simulation bereitgestellt werden können. Dies wird dann verwendet, um die analogen Parameter der vorgeschlagenen FPAA-Struktur zu untersuchen. Zum Schluss werden Simulationen eines komplexen Filteraufbaus auf Systemebene betrachtet, um die Fähigkeiten des Systems aufzuzeigen.

\section{Konfigurationsdaten}

In den CABs befinden sich Register, in denen die Konfigurationsdaten des Chips gespeichert werden. Für die digitale Rekonfiguration durch einen externen Mikrocontroller oder ein Gatearray wird durch eine globale Logik im Analogarray ein Kommunikationsprotokoll implementiert. Die Konfigurationsdaten eines CABs setzen sich aus 7 Zweigen zusammen, wobei jeder Zweig 14 Bit zur Speicherung des Schaltzustandes der Einheitstranskonduktoren besitzt. Um die Zustände aller Konfigurationsschalter des analogen Arrays vollständig festzulegen, wird daher eine Folge von 1666 Bits benötigt. Da ohne die korrekte Konfiguration der Register die Funktionsweise des Analogteils nicht genau definiert ist, kann weder eine Simulation berechnet noch der reale Chip betrieben werden, wenn an die Register keine Konfigurationsdaten angelegt wurden. Zur vollständigen Ausschöpfung der Möglichkeiten des Analogarrays soll der Designer in der Lage sein, viele verschiedene Einstellungen ausprobieren und den kompletten Satz von 1666 Bits nutzen zu können, um eine Konfiguration zusammenzustellen. Für diese Daten muss eine Darstellung gefunden werden, die das intuitive Verständnis der Konfiguration fördert und eine schnelle und einfache Eingabe von verschiedenen Filterstrukturen erlaubt.

\subsection{Graphische Eingabe des Entwurfs}

Es wurde eine graphische Benutzerschnittstelle (GUI) gewählt, welche den Chip auf Systemebene mit 17 CABs und 8 IO-Buffern wie in Abb. 1 darstellt. Die Zwischenverbindungen sind entweder invertierend oder nicht-invertierend und werden durch Pfeile visualisiert. Die Transkonduktanz jeden Zweiges wird im Körper des CABs angegeben. Besonders nützlich an dieser Sicht ist, dass der direkte Vergleich mit schematischen Blockdiagrammen, die oft zum Beschreiben von $\mathrm{g}_{m}$-C-Filtern verwendet werden, leicht möglich ist. Änderungen der Konfiguration werden an einem Computer direkt in die GUI eingegeben und die Daten werden in einer Bitdatei, die für jeden Konfigurationsschalter die binäre Einstellung enthält, gespeichert.

Die GUI und die Bitdatei wurden so konstruiert, dass sie eine gemeinsame Basis für Simulation und Chipkonfiguration bilden. Dieselbe GUI ist sowohl fähig, ein kommerzielles Simulationswerkzeug mit einer ausgewählten Konfiguration zu starten als auch einen realen Chip über USB und Boundary-Scan zu programmieren. Dies beinhaltet, dass der reale Chip mit einer digitalen Logik ausgestattet ist, die das Laden einer Bitdatei und die sofortige Instantiierung eines Filters in Hardware erlaubt, sobald eine Struktur durch Simulation verifiziert wurde.

\subsection{Laden der Konfigurationsdaten für die Simulation}

Bei der Erstellung des Arrays wird ein einzelner Einheitstranskonduktor zur Dimensionierung des Schaltkreises simuliert. Für eine Charakterisierung des Transkonduktors im System ist es jedoch notwendig ihn zu untersuchen, wenn er in das komplette Array eingebettet ist, um den Einfluss von Verbindungen zu Nachbarzellen und parasitären Effekten auszuwerten. Wie bereits erwähnt muss zu diesem Zweck eine Konfiguration in der GUI erstellt werden und in die Simulation geladen werden, damit die Spannungsquellen entsprechend der Schaltzustände instantiiert werden können.

Das Laden einer Bitdatei wird durch ein AHDL-Modul erreicht, welches am Anfang einer Simulation einmal ausgeführt wird. Während jeden Durchlaufs ist somit die Konfiguration fest, so wie es für die Berechnung einer Übertragungsfunktion notwendig ist. Wegen des engen Zusammenspiels zwischen der maßgeschneiderten GUI und den kommerziellen Simulationswerkzeugen kann die Netzliste des Analogarrays unabhängig von der des Konfigurationsladers erstellt werden. Sobald die Netzliste erstellt ist, liest der Konfigurationslader die Bitdatei und setzt entsprechend die Spannungen. Solange sich das Design des analogen Arrays nicht ändert, ist eine zusätzliche Netzlistenerstellung nicht nötig um die Simulation mit einer anderen Konfiguration laufen zu lassen. So werden unnötige Berechnungen vermieden.

\section{Simulationsergebnisse von elementaren Filtern}

Zur Charakterisierung des analog Arrays wird der Frequenzgang mit einem Model auf Transistorebene simuliert und die Parameter des Phasen/Betrags-Graphen extrahiert. Diese Pa- 


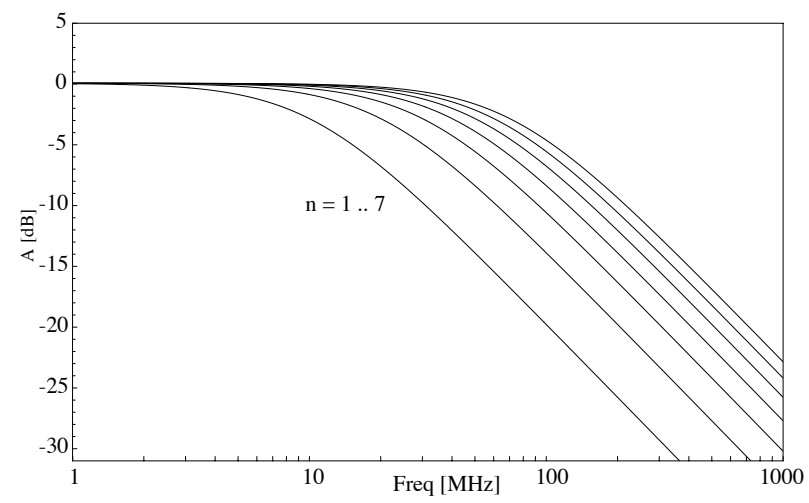

Abbildung 2. Übertragungsfunktion eines Zweiges für $n=1 \ldots 7$.

rameter erlauben einen Vergleich mit theoretischen Erwartungen und die Beurteilung von verschiedenen Filterstrukturen. Wie in (Becker and Manoli, 2004a) dargestellt, wurde eine $0.18 \mu \mathrm{m}$ CMOS Technologie für die Fertigung des FPAA ausgewählt und die BSIM3v3-Modelle des dazugehörenden Design-Kits verwendet.

Ein Vorteil des vorgestellten Systems ist wie bereits erwähnt, dass die Parameter der Schaltung im kommerziellen Schaltplaneditor verändert werden können. Verschiedene Designs der Einheitstranskonduktoren können so implementiert und bei der Simulation verwendet werden. Das erstellte Simulationssystem bindet die entsprechende Einheitszellen in die Chipstruktur ein und simuliert den gesamten Chip auf Transistorebene. Auf diese Art und Weise besteht die Möglichkeit verschiedene Transkonduktordesigns sehr einfach zu vergleichen.

\subsection{Eine einzelne $\mathrm{g}_{m}-\mathrm{C}-\mathrm{Stufe}$}

Zur Verifikation des vorgeschlagenen Konzepts muss bewiesen werden, dass sich eine einzelne $\mathrm{g}_{m}$-C-Stufe im Einklang mit der theoretischen Übertragungsfunktion befindet. Die Übertragungsfunktion $H(s)$ einer einzelnen $\mathrm{g}_{m}$-C-Stufe ist ideal durch die Einpol-Gleichung (1) gegeben, wobei $A_{0}$ der Verstärkungsparameter und $f_{c}$ die Eckfrequenz ist.

$H(s)=\frac{A_{0}}{1+\frac{s}{2 \pi f_{c}}}$

Da der Transkonduktor für einen Zweig über die Anzahl der Einheits- $g_{m} \mathrm{~s}$, die parallel geschaltet sind, einstellbar ist, hängt die Eckfrequenz von der Anzahl $n$ linear ab. Durch Simulation erhält man folgende Ergebnisse:

\begin{tabular}{c|c|c|c|c|c|c|c}
$\mathrm{n}$ & 1 & 2 & 3 & 4 & 5 & 6 & 7 \\
\hline$f_{c}[\mathrm{MHz}]$ & 10.05 & 20.12 & 30.19 & 40.27 & 50.36 & 60.45 & 70.55
\end{tabular}

An diese Zahlen wurde die Übertragungsfunktion eines Einpols angenähert, so dass die Abhängigkeit von $n$ berechnet werden kann.

$f_{c}(n)=n \cdot 10.07 \mathrm{MHz} ; \quad A_{0}=0.997$

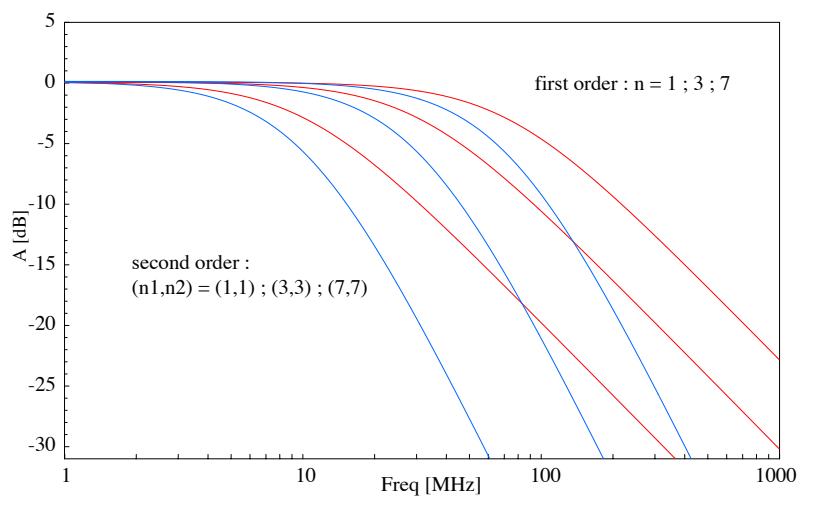

Abbildung 3. Übertragungsfunktion 1. und 2. Ordnung.

Die lineare Abhängigkeit der Eckfrequenz $f_{c}$ vom Schaltparameter $n$ und die konstante Verstärkung $A_{0}$ sind in Abb. (2) dargestellt. Beide Gleichungen zeigen eine ideale Übereinstimmung mit der Theorie; der Fehler ist vernachlässigbar.

In Abb. 2 sind mit $n=1 \ldots 7$ simulierte Übertragungsfunktionen aufgezeichnet. Dies zeigt die Programmierbarkeit der analogen Eckfrequenz durch ein $n$-repräsentierendes digitales Wort und damit den Schlüssel zur Instantiierung von rekonfigurierbaren adaptiven Filtern auf dem Array. Die Graphen zeigen die erwünschte lineare Verschiebung der Eckfrequenz in Abhängigkeit der Anzahl der aktivierten $g_{m}$-Zellen. Die Steigung zeigt die charakteristische Dämpfung von -20 dB pro Dekade. Die theoretischen Vorhersagen des idealen Einpols und die Simulationsergebnisse sind deckungsgleich.

\subsection{Serienschaltung von zwei $\mathrm{g}_{m}-\mathrm{C}$ Stufen}

Nachdem die Funktionalität einer einzelnen $\mathrm{g}_{m}$-C Stufe als Tiefpassfilter erster Ordnung charakterisiert wurde, ist als nächstes die Serienschaltung von zwei $\mathrm{g}_{m}-\mathrm{C}$ Stufen zu untersuchen. Die ideale Übertragungsfunktion $H(s)$ für einen Tiefpassfilter zweiter Ordnung ist:

$H(s)=\frac{A_{0}^{2}}{\left(1+\frac{s}{2 \pi n_{1} f_{c}}\right) \cdot\left(1+\frac{s}{2 \pi n_{2} f_{c}}\right)}$

$A_{0}$ ist wieder der Verstärkungsfaktor und $n_{1} f_{c}$ und $n_{2} f_{c}$ die Eckfrequenzen der Einzelstufen. Die Übertragungsfunktion des Zweipol-Systems erhält man durch die Substitution der angepassten Parameter des Einpols aus dem vorherigen Abschnitt. Für einen Tiefpass-Filter zweiter Ordnung sollte die Steigung der Übertragungsfunktion doppelt so groß wie beim System erster Ordnung sein. Bei einer Zelle mit $A_{0} \approx 1$ sollte der quadrierte Verstärkungsfaktor die Übertragungsfunktion nicht signifikant beeinflussen.

Abbildung 3 vergleicht einen einstellbaren Filters erster Ordnung mit $n=1 ; 3 ; 7$ und einem Filter zweiter Ordnung 


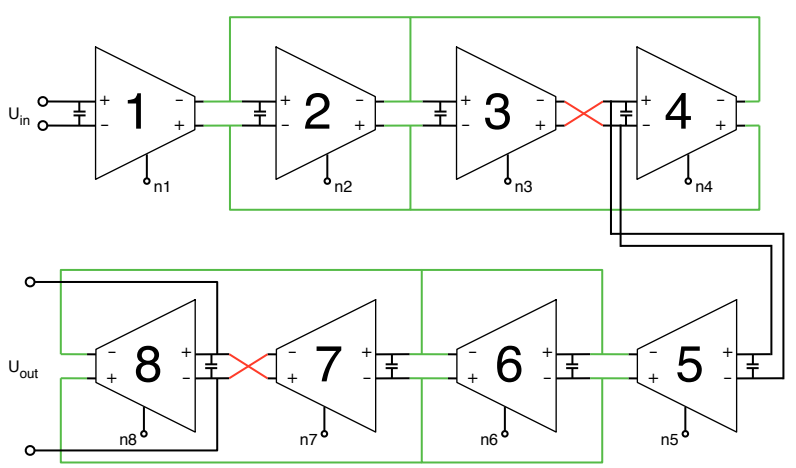

Abbildung 4. Schaltbild eines Butterworth biquad band-pass Filters vierter Ordnung.

mit $(n 1, n 2)=(1,1) ;(3,3) ;(7,7)$. Die Simulationsergebnisse decken sich wieder perfekt mit den theoretischen Vorhersagen und zeigen die erwarteten Eigenschaften. Dies ist ein bemerkenswertes Ergebnis, da es verifiziert, dass eine Serienschaltung von zwei $\mathrm{g}_{m}$-C Stufen im vorgeschlagenen Array sich exakt so verhält, wie von der Systemtheorie vorhergesagt. Übertragungsfunktionen komplexer Filterstrukturen können folglich durch eine Zusammensetzung von parallelen und seriellen Verbindungen von Einzelstufen berechnet werden. Dies dient als Basis für die Instantiierung von Filterstrukturen auf dem Array und zur Vorhersage ihrer Übertragungsfunktionen.

\section{Simulation von komplexen Filterstrukturen}

Unter Benutzung dieser Basischarakteristika ist es möglich, Übertragungsfunktionen für komplexere Filterstrukturen theoretisch zu berechnen. Ein Teil der aktuell laufenden Forschung ist die Charakterisierung von einfachen Schaltungen, um eine Sammlung von Bauelementen für größere Designs zu erstellen. Interessante Eigenschaften des Analogarrays ergeben sich daraus, dass passive Filterkomponeten durch aktive $g_{m}$-Zellen emuliert werden können wie zum Beispiel die Gyratorschaltung. In diesem Zusammenhang sollen jedoch die Möglichkeiten des Analogarrays und des Simulationssystems durch das Simulieren einer anspruchsvolleren Struktur dargestellt werden. Da üblicherweise Implementierungen von Butterworthfiltern benutzt werden, die Leistungsfähigkeit eines Design zu zeigen, wird im Folgenden ein Butterworth Bandpassfilter vierter Ordnung in Biquadrealisierung vorgestellt.

Abbildung 4 zeigt das Blockdiagramm einer in Pavan and Tsividis (2000) benutzten Filterstruktur als festen Aufbau. Für das FPAA ist dies allerdings nur eine von vielen Konfigurationsmöglichkeiten. Unter Einhaltung der selben Struktur sind offensichtlich alle Einzeltranskonduktanzen einstellbar. Wie aus der Systemtheorie abgeleitet werden

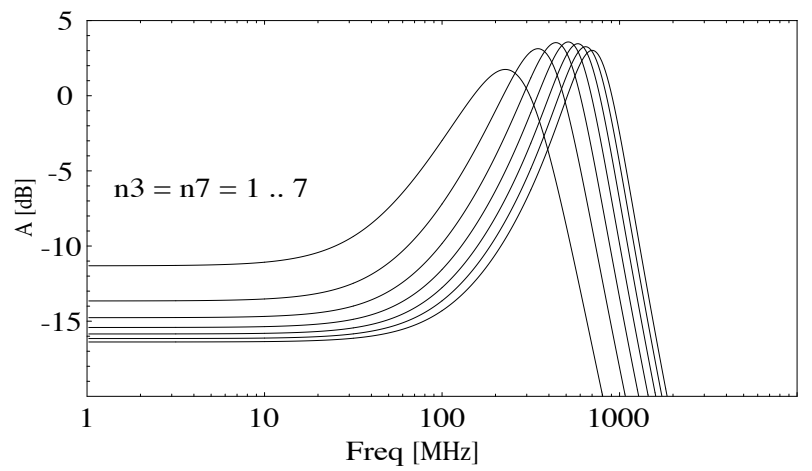

Abbildung 5. Übertragungsfunktion eines Butterworth-Filters mit Parameterverlauf.

kann, kontrollieren die Einzeltranskonduktanzen verschiedene Parameter des Filters. Hier können allerdings nicht alle Abhängigkeiten dieser 8 Parameter ausgewertet werden. Eine der interessantesten Einstellmöglichkeiten ist in Abb. 5 dargestellt. Es zeigt die simulierten Übertragungsfunktionen des simulierten Filters für die Parameterzusammenstellung $(7,1, n 3,7,7,1, n 7,7)$ und einen Durchlauf von $(n 3=n 7=1 \ldots 7)$. Man kann sehen, dass die Konfiguration wie erwartet einen Bandpassfilter mit einer digital einstellbaren Mittenfrequenz repräsentiert.

Diese Simulationsergebnisse wurden abermals durch die Entwicklungsumgebung gewonnen, indem das Blockdiagramm (Abb. 4) in die GUI des Eingabewerkzeugs (Abb. 1) eingegeben wurde. Die Farben der Verbindungen wurden beibehalten, um den Vergleich der Abbildung des Blockdiagramms und der GUI zu fördern. Dasselbe GUI steuert dann ein kommerzielles Simulationswerkzeug mit den BSIM3v3Transistormodellen und der Struktur des analogen Arrays.

\section{Zusammenfassung}

Es wurde eine Designsystem vorgestellt, das eine einfache Eingabe von Konfigurationsdaten für programmierbare Analogarrays ermöglicht. Ein graphisches Benutzerinterface speichert eine gewählte Konfiguration in einer Bitdatei und steuert die Skript-Schnittstelle eines kommerziellen Simulationswerkzeuges an, um eine Simulation des gesamten Analogarrays auf Transistorebene auszuführen. Die Initialisierung der Simulation mit den Konfigurationsdaten wird über ein AHDL-Modell durchgeführt, welches die Bitdatei lädt und die benötigten Spannungsquellen entsprechend setzt.

Es wird die Anwendung dieses Systems auf eine vorgeschlagene FPAA-Methodik gezeigt, die eine intuitive Konfiguration von $\mathrm{g}_{m}$-C-Filter Strukturen erlaubt. Beim Design des Analogarrays wurden verschiedene Filterstrukturen simuliert. Das in (Becker and Manoli, 2004a) vorgeschlagene Arbeitsprinzip wurde damit durch Simulation bestätigt. 
Schließlich wurde die Umsetzungen von zeitkontinuierlichen aktiven Filtern hoher Frequenz auf dem FPAA durch die exemplarische Instantiierung und Simulation eines Butterworth Filters vierter Ordnung gezeigt. Die Charakterisierung von einfachen Schaltungen für eine Sammlung von elementaren Bauelementen ist ein Teil der aktuellen Forschung.

Letztlich ist die Fertigung des Chips geplant, um die Simulationsergebnisse mit Hardware zu vergleichen und die Übertragungsfunktion des realen Chips zu charakterisieren. Dann ist es möglich, ein System zu erhalten innerhalb dessen analoge Filter aus vordefinierten Bausteinen zusammengesetzt, mit Simulationen auf Transistorebene verifiziert und auf Hardware instantiiert werden können.

\section{Literatur}

Becker, J. and Manoli, Y.: A new architecture of field programmable analog arrays for reconfigurable instantiation of continuous-time filters, IEEE Intern. Conf. on Field Programmable Techn. (ICFPT), 2004a.

Becker, J. and Manoli, Y.: A Continuous-Time Field Programmable Analog Array (FPAA) consisting of digitally reconfigurable $\mathrm{G}_{m}$-cells, IEEE Intern. Symp. on Circuits and Systems (ISCAS), 2004b.

Pavan, S. and Tsividis, Y.: High Frequency Continuous Time Filters in Digital CMOS Processes, Kluwer Academic Publishers, Boston, 2000. 